\title{
EM BUSCA DO ESTADO DA ARTE NA GERAÇÃO DE CONHECIMENTOS GEOSSISTÊMICOS PARA A CIÊNCIA DO SOLO
}

\author{
Thamyres Sabrina Gonçalves ${ }^{1 *}$ Alexandre Christófaro Silva ${ }^{2}$
}

\footnotetext{
${ }^{1}$ Geógrafa, Mestre em Ciência Florestal, Doutoranda em Produção Vegetal na Área de Manejo e Conservação de Solos, Universidade Federal dos Vales do Jequitinhonha e Mucuri, Diamantina - MG. *E-mail do autor correspondente: sabrina5thamy@yahoo.com.br

${ }^{2}$ Docente da Universidade Federal dos Vales do Jequitinhonha e Mucuri, Diamantina - MG.
}

RESUMO: O ecossistema solo é amplamente divulgado como sendo um dos mais ricos ecossistemas do mundo, todavia, apesar de ser reconhecida essa grande complexidade ecossitêmica do solo, pouco se conhece acerca de suas interações. Das interações que são conhecidas, a maior parte delas não foi estudada dentro da dinâmica natural de funcionamento do ecossistema do solo. Portanto, são amplamente necessários trabalhos que investiguem o estado da arte do conhecimento em ecologia do solo, biologia do solo e de todas as interações que acontecem nesse ecossistema, pois somente a partir do conhecimento de onde estamos, em termos de conhecimento científico sobre esses processos de interação no solo, abaixo e acima da camada superficial, é que poderemos avançar de modo a produzir um conhecimento que possa atender as demandas acerca dos processos ecológicos no solo. Os resultados dessa revisão mostram que houve importantes avanços na ciência acerca dos processos de interação ecológica no solo que em muito contribuíram para o manejo e conservação de ecossistemas naturais e agrícolas, mas que ainda existem muitos assuntos e organismos pouco ou quase nada estudados. Conclui-se que o ecossistema solo pode ser o ambiente chave para a compreensão de como os processos antrópicos têm influenciado a dinâmica de uso e ocupação de solos no planeta e também de como poderemos fazer para tentar recuperar os processos biológicos que foram perdidos em alguns ecossistemas.

Palavras-chave: Biologia do solo. Ecossistema edáfico. Interações multitróficas.

\section{IN SEARCH OF THE STATE OF ART IN GENERATING GEOSYSTEMS KNOWLEDGE FOR SOIL SCIENCE}

\begin{abstract}
The soil ecosystem is widely reported as being one of the richest ecosystems in the world however, despite being recognized this great ecosystemic complexity Soil little is known about the interactions that occur in this ecosystem. The interactions that are known not been studied within the natural dynamics of functioning of the soil ecosystem. So, are widely needed studies that investigate the state of the art knowledge on soil ecology, soil biology and all the interactions that take place in this ecosystem, because only from the knowledge of where we are in terms of scientific knowledge about these processes interaction in the ground, below and above the surface layer soil organisms is that we can
\end{abstract}


move forward in order to produce knowledge that can meet the demands on the ecological processes in soil. The results of this literature review shows that there have been important advances in science about the processes of ecological interactions in the soil ecosystem and contributed greatly to the ecology, but there are still many issues and little or nothing organisms studied. It was concluded that the soil ecosystem can be an environment of great contributions to the understanding of how the anthropogenic processes have influenced the Earth's biodiversity and also how we can do to try to recover the biological processes that have been lost in some ecosystems.

Key words: Biology of soil. Edaphic ecosystem. Multitrophic interactions.

\section{INTRODUÇÃO}

Desde que está presente na terra, o ser humano se preocupa em estudar e conhecer melhor sobre os solos, suas cores, sua fertilidade natural, condições físicas e produtividade agrícola (LEPSCH, 2002). Todavia os processos biológicos e as interações ecológicas não estiveram desde sempre dentre as prioridades no estudo dos solos (LEPSCH, 2002). Apesar disso, o conceito de fertilidade de solo desde o princípio da civilização do século I ao $\mathrm{X}$ a concepção de solo fértil baseava-se na integração das fases biológica, física e química do solo, em contínua renovação pelo cultivo com técnicas apropriadas, ou melhor dizendo, na capacidade natural de resiliência do solo frente aos processos de modificação ao longo do tempo.

Além disso, há de se pensar que o estudo de interações ecológicas em qualquer tipo de ecossistema, depende do nível e da situação do conhecimento existente e disponível. Para Lepsch (2002), estudos de solo não se desenvolvem com profissionais de apenas uma área, pois prescindem de uma integração multiprofissional e principalmente entre diferentes áreas do conhecimento científico.

Enquanto a ciência do solo se dedicou muito mais aos processos de interpretação da fertilidade dos solos e suas influências na produtividade agrícola, a ecologia se ocupou primeiro de conhecer melhor as interações que ocorriam acima do solo. Isso não quer dizer que não se reconhecia a importância ecológica do solo e suas influências nas interações que ocorriam acima deste, mas sim que há uma maior quantidade de investigações científicas sobre as interações acima do solo do que nas camadas edáficas em si e no subsolo.

Isso pode ser compreendido se considerarmos as questões metodológicas que norteiam as pesquisas em ecologia de ecossistemas, pois, acima do solo, estas interações são geralmente mais visíveis, ou seja, mais facilmente mensuráveis, embora haja certamente exceções.

O trabalho de McPhee e Aarssen (2001) faz uma revisão que em muito contribui nessa discussão, onde os autores avaliam diversas metodologias de estudos, de interações entre plantas abaixo e acima do solo, abordando sobre as possibilidades e limitações de cada uma 
destas, apontando que todas as metodologias estudadas apresentam aspectos positivos e negativos em relação ao estudo de interações em cada um dos níveis tróficos (abaixo e acima do solo), e que a principal dificuldade na compreensão integrada das interações se deve ao fato de que os métodos de pesquisa geralmente subdividem o ecossistema em: abaixo do solo, e acima do solo, não investigando na maioria das vezes as interações multitróficas que existem ao se considerar os processos de maneira integrada.

$\mathrm{Na}$ teoria essa proposta de integração é muito pertinente, e de fato deveria ocorrer ao menos com mais frequência na pesquisa científica, pois é provavelmente impossível medir todas as interações que ocorrem com uma única espécie abaixo e acima do solo, mas existe sim a possibilidade de integração de conhecimentos advindos de outros estudos que possam contribuir na compreensão da interação mútua entre o que ocorre com as espécies abaixo e acima do solo se forem considerados estudos multidisciplinares.

De fato a questão teórico-metodológica parece ser um dos principais entraves da análise integrada das interações abaixo e acima do solo, pois nos estudos acima do solo encontram-se atualmente fortes discussões acerca de conceitos como nicho, comunidade, espécie, entre outros. Já nos trabalhos que abordam interações abaixo do solo, embora essas discussões teóricas supracitadas também influenciem na ecologia do que acontece abaixo do solo, os problemas mais citados pela maior parte dos pesquisadores como sendo a principal dificuldade da pesquisa no ecossistema solo e subsolo, são sempre relacionadas as metodologias, que não atendem a todos os grupos de organismos presentes no solo, que não identificam associações entre esses organismos a nível inter e intraespecífico e que não são sempre adaptáveis ou mesmo acessíveis para diferentes ecossistemas.

No Brasil, o número de trabalhos sobre os organismos do solo é ínfimo diante da diversidade de ecossistemas do país e do desconhecimento da própria biodiversidade do solo. No debate sobre a conservação dos recursos naturais, o solo nem sempre tem a atenção necessária para que as ações de conservação sejam efetivas. Por outro lado a degradação dos solos está de certo modo na base de todo o problema da perda cada vez maior de biodiversidade no Brasil. Portanto, esse trabalho tem o objetivo de fazer uma revisão do estado da arte no conhecimento sobre as interações ecológicas que ocorrem abaixo e acima do solo de uma perspectiva conservacionista, avaliando o esforço da pesquisa, as temáticas abordadas, as contribuições desses trabalhos para a ciência do solo e as perspectivas atuais e futuras dessas pesquisas.

\section{REVISÃO BIBLIOGRÁFICA}

\section{O estado da arte na pesquisa sobre as interações abaixo e acima do solo de forma integrada}

Em uma busca com o tema organismos do solo, na página eletrônica do Google Acadêmico, que é à base de dados mais abrangente disponível de forma gratuita e simples (MUGNAINI e STREHL, 2008), nota-se que a maior parte dos trabalhos fala da dificuldade 
metodológica em se coletar e identificar os organismos que vivem no solo, demostrando a importância e a necessidade de novos estudos na área de taxonomia e sistemática desses grupos (GIRACCA et al., 2003; ZILLI et al.,2003; MOÇO et al., 2005; ANTONIOLLI et al., 2006; BARETTA et al., 2008; ROVEDDER et al., 2009; VAZ DE MELO et al., 2009).

Em um levantamento na Revista Brasileira de Ciência do Solo (RBCS) (Fig.1), percebe-se uma grande variação na quantidade de publicações sobre biologia do solo entre o período de 2003 a 2014, o que provavelmente tem a ver com o momento da pesquisa científica em cada época e as demandas da sociedade que influenciam nos órgãos e agências de fomento que elaboram editais para financiamento de projetos de pesquisa científica.

Neste levantamento, que considerou apenas a seção de biologia do solo, e os trabalhos realizados em ecossistemas naturais ou em experimentos com espécies nativas foram encontrados 32 publicações, sendo os anos que apresentaram maior quantidade de publicações 2004, 2008 e 2010. Em alguns anos como os de 2005 a 2007, houve uma queda na quantidade de publicações, o que mostra que não existe no Brasil um esforço contínuo na produção de conhecimento científico sobre as interações no solo.

Já no período de 2011 a 2014 não foi encontrado nenhum trabalho, isso porque a própria seção de biologia do solo praticamente deixou de existir, assim como muitas outras seções na revista que se relacionavam as demais áreas temáticas da ciência do solo, pois, todos os trabalhos foram nesse período enquadrados nas seções de processos do solo no espaço e no tempo, e na área de manejo do solo que engloba a maior parte das publicações atualmente. De fato, o momento atual da ciência do solo é o das técnicas de manejo, da diversificação de sistemas, do desenvolvimento de novas formas de aportar matéria orgânica no solo, o que é fomentado, sobretudo pelo interesse no estoque de carbono no solo para mitigação do efeito das mudanças climáticas.

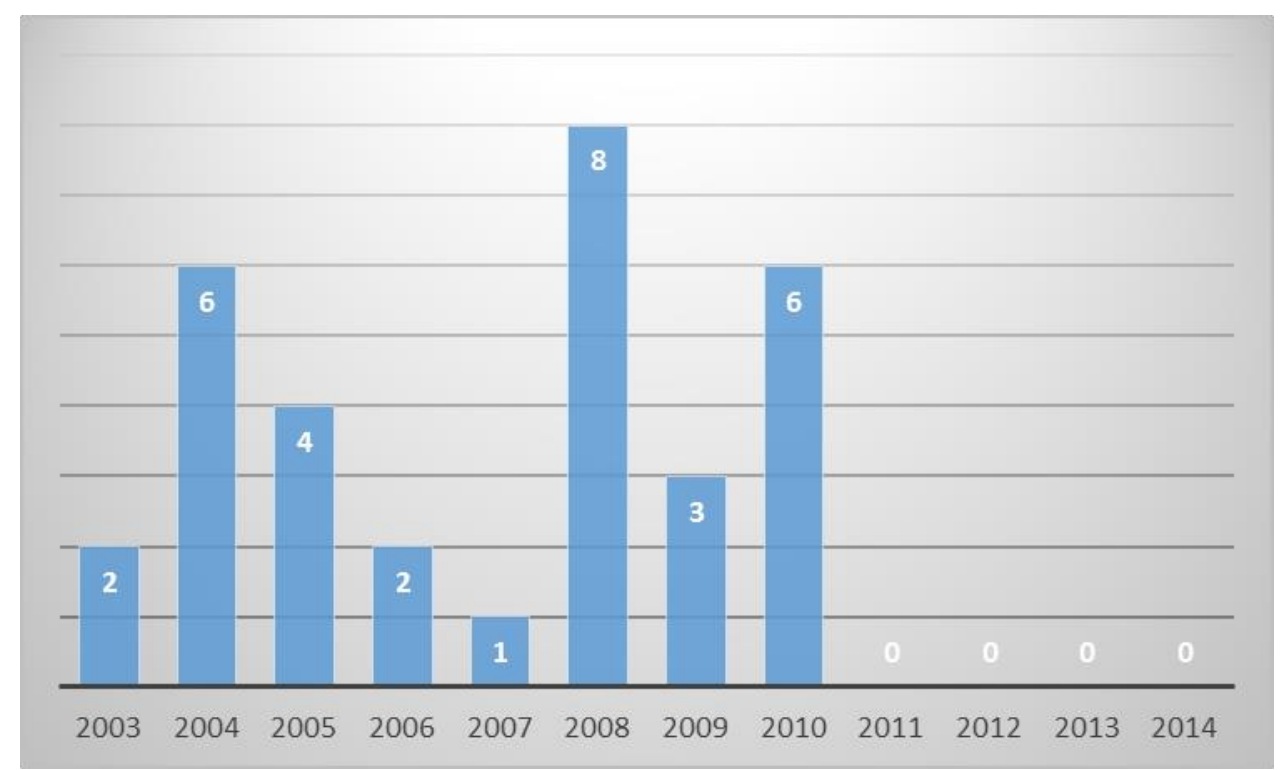

Figura 1. Quantidade de publicações na Revista Brasileira de Ciência do Solo sobre interações ecológicas no ambiente edáfico na área de Biologia do Solo entre 2003 e 2014.

Cultura Agronômica, Ilha Solteira, v.27, n.2, p.205-216, 2018 
Mas é importante destacar que, muitos dos trabalhos publicados em outras áreas que não a biologia do solo podem trazer contribuições importantes sobre o conhecimento das interações ecológicas no solo, inclusive estudos experimentais realizados com plantas exóticas cultivadas.

Apesar de haverem muitas limitações na transposição de um conhecimento estritamente agrícola para os ecossistemas naturais, existem importantes conexões de processos no sistema solo-planta que podem ser mais bem compreendidos ao integrar-se a pesquisa sobre como os ecossistemas agrícolas afetam as vegetações nativas e vice versa, podendo ser estas informações imprescindíveis ao manejo conservacionista de solos e culturas.

Outro aspecto a ser considerado é de que por estar a ciência do solo, historicamente vinculada à produção vegetal, tanto os conceitos dessa ciência quanto suas pesquisas, hipóteses e experimentos na maioria das vezes não objetivam conhecer sobre o funcionamento do solo enquanto um ecossistema, mas sim como um agrossistema, o que traz a necessidade de aprofundamento em discussões tanto teóricas quanto metodológicas na pesquisa com foco em conservação de ecossistemas. Nesse sentido já é possível encontrar trabalhos que trazem relevantes contribuições como Moreira et al. (2013).

Isso tem melhorado muito nos últimos anos, porque a grande demanda da agricultura de baixo carbono tem como base essencial para o seu desenvolvimento o aporte de matéria orgânica no solo (VEZZANI e MIELNICZUK, 2009) que por sua vez favorece as interações entre os organismos do solo gerando maior quantidade de hábitats e mais biodiversidade.

Uma das maiores instituições de pesquisa no Brasil, na área de solos, é a Empresa Brasileira de Pesquisa Agropecuária (EMBRAPA). Em uma busca no site desta instituição do período de 2010 a 2014, com a frase interações ecológicas no solo, encontra-se 12.656 resultados de publicações, e com above-bellow ground interactions, encontra-se 80 resultados que incluem todo o tipo de publicações, desde resumos a trabalhos completos de anais de eventos, livros, cartilhas e boletins técnico-científicos.

Em uma análise quantitativa de publicações ao longo dos últimos anos no período de 2001 a 2014 (Figura 2), nota-se um crescimento significativo no número de resultados, o que demonstra ter aumentado, por parte da Embrapa, o esforço para pesquisa sobre a atividade biológica no solo. Considerando que a instituição possui a maior parte de suas pesquisas destinadas a produzir conhecimento aplicável para o setor agropecuário brasileiro, isso significa alguns avanços na sustentabilidade agrícola do país desde que este conhecimento publicado esteja chegando de fato ao produtor rural no campo. Mas, para construção de um conhecimento integrado acerca das interações no solo, tanto abaixo quanto acima da camada superficial, é preciso que os pesquisadores se esforcem para o desenvolvimento de mais estudos nesse sentido.

Cultura Agronômica, Ilha Solteira, v.27, n.2, p.205-216, 2018 


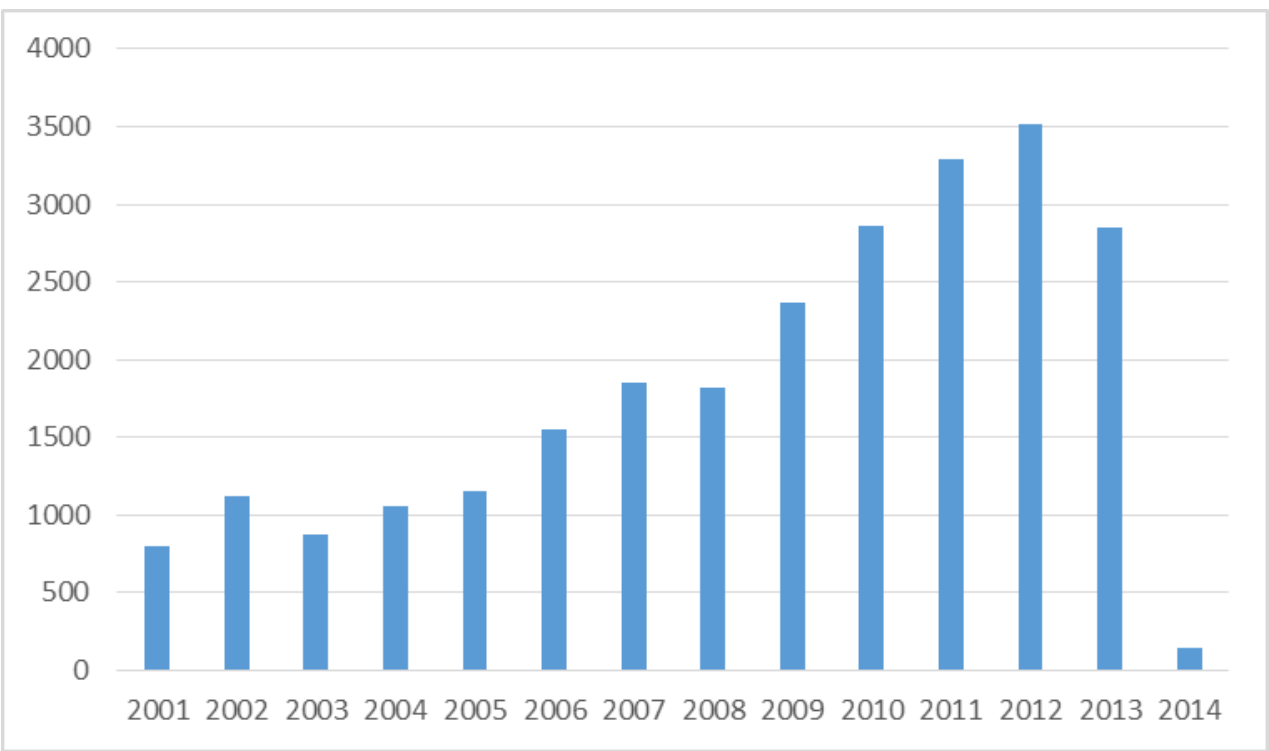

Figura 2. Publicações da Empresa Brasileira de Pesquisa Agropecuária sobre interações ecológicas no solo entre 2001 e 2014.

Estudos sobre os processos de interação abaixo e acima do solo intensificaram-se mundialmente nas últimas três décadas aproximadamente. Uma das mais extensas publicações sobre os processos de interação abaixo e acima do solo é o livro editado por Persson (1990) que condensa quase trezentas páginas com resultados de pesquisas sobre a inter-relação entre as interações abaixo e acima do solo em ambientes com solos acidificados.

O trabalho de Singh et al. (1989) fala da influência das interações abaixo e acima do solo em cultivos agrícolas no semiárido indiano. Mais de vinte anos após esta publicação, Vasconcelos et al. (2012) resgatam uma importante metodologia de cultivo agrícola tradicionalmente empregada em regiões tropicais da África e Ásia, trazendo a importância da incorporação de adubo verde as propriedades de agricultura familiar no semiárido do nordeste brasileiro, através da integração de conhecimentos entre o manejo do solo e de espécies arbóreas de pequeno e médio porte fixadoras de nitrogênio, fazendo cultivo em aléias.

Soler et al. (2005) trazem uma contribuição sobre o estudo das interações abaixo e acima do solo de maneira integrada, englobando relações multitróficas, pois estes autores avaliaram se a herbivoria no sistema radicular influencia no desempenho da planta, dos parasitoides, hiperparasitóides e seus inimigos naturais, encontrando resultados positivos que comprovaram que a interação entre o inseto herbívoro e o sistema radicular da planta atuavam no crescimento desta e controle populacional dos organismos associados a espécie acima do solo. Ainda Wackers e Bezemer (2003), verificaram que a herbivoria na raiz da planta pode levá-la ao desenvolvimento de defesas indiretas como a produção de néctar extrafloral, mostrando que os herbívoros que se alimentam de raízes podem alterar defesas das plantas acima do solo.

Cultura Agronômica, Ilha Solteira, v.27, n.2, p.205-216, 2018 
Blomqvist et al. (2000) estudaram as inter-relações entre interações abaixo e acima do solo em um ambiente de pastagem, e concluíram que as interações abaixo do solo formam micro hábitats na paisagem que correspondem aos mosaicos na vegetação. Baretta et al. (2008) verificaram que a interação de um grupo de organismos do solo com espécies arbóreas dominantes em seu hábitat natural, fez com que a pressão antrópica sobre a planta diminuísse suas populações ocasionando uma diminuição na biodiversidade de organismos do solo associados a essas espécies.

Rovedder et al. (2009) fizeram um trabalho avaliando a eficácia da utilização de plantas nativas na recuperação de solos degradados por arenização e constataram que os organismos do solo possuem interações tróficas mais fortes com as espécies nativas e que a grande utilização de plantas exóticas na recuperação de áreas degradadas muitas vezes pode alterar a atividade biológica dos organismos do solo, porque as plantas nativas atuam na modificação da estrutura física e química do solo em uma intensidade e funcionalidade em que os organismos do solo conseguem acompanhar e se adaptar evolutiva e comportamentalmente a essas mudanças.

O trabalho de Fukami et al. (2006) aborda o efeito em cascata da introdução de espécies exóticas na diminuição da fertilidade do solo e suas influências no crescimento e desenvolvimento da vegetação. Em ecossistemas naturais, o efeito cascata advindo dos processos de fragmentação florestal está no cerne das discussões sobre a conservação da flora nativa do Brasil, já que esse efeito tende a se acentuar com a degradação dos ecossistemas.

Após décadas de pesquisa, Wardle (2006) conclui que são as interações bióticas que regulam a biodiversidade no solo, o que é admitido também pela maioria dos trabalhos supracitados. Mas os fatores abióticos são muitas vezes determinantes nas condições ambientais que irão induzir as espécies a interagirem (FIGUEIREDO et al., 2008), portanto não se pode apontar singularmente fatores bióticos ou abióticos como mecanismo exclusivo na manutenção da biodiversidade do solo.

Os trabalhos atuais têm abordado estudos com interações mais complexas envolvendo mais espécies, fatores ambientais e níveis tróficos. Nesse contexto, Heil (2011) discute que além dos vários fatores não mensuráveis nas inter-relações, entre interação abaixo e acima do solo, há muitos processos evolutivos atuando com intensidades desconhecidas em cada organismo envolvido na interação, e que os avanços nos estudos de fisiologia das espécies associados aos de filogenética são necessários para amenizar incongruências entre o que podemos e o que não podemos medir dessas interações abaixo e acima do solo.

Pizano et al. (2011) avaliaram a influência das interações acima e abaixo do solo de maneira integrada na preferência de algumas espécies arbóreas por diferentes tipos de hábitats. Stevnbak et al. (2012) correlacionou as interações acima e abaixo do solo com os efeitos das mudanças climáticas. Born et al. (2013) avaliou a relação entre diferentes espécies de um mesmo gênero de plantas especialistas e generalistas com diferentes fatores

Cultura Agronômica, Ilha Solteira, v.27, n.2, p.205-216, 2018 
abióticos como saturação hídrica, disponibilidade de luz e variações pedogeomorfológicas ao longo das diferentes fases de desenvolvimento da planta, constatando que as espécies possuem adaptações a determinados ambientes que foram adquiridas ao longo da história de vida da espécie.

Figueiredo et al. (2008) defende a ação dos microorganismos do solo para promoção de uma agricultura mais sustentável e maior compreensão dos processos ecológicos sobre a biodiversidade no solo abordando diferentes assuntos como a fixação biológica de nitrogênio, a influência dos microorganismos no crescimento das plantas, componentes orgânicos do solo, biofertilizantes e recuperação de áreas degradadas, trazendo resultados que podem auxiliar na conservação do solo.

No Brasil as pesquisas sobre a biologia do solo se concentram no estudo de microorganismos (SILVA et al., 2010; GIONGO et al., 2010; MARTINS et al., 2010), principalmente a biomassa microbiana que tem sido o principal componente utilizado para avaliar a qualidade e a atividade biológica do solo (ZILLI et al., 2003), isso devido a grande evolução científica pela qual tem passado os estudos com marcadores moleculares (FIGUEIREDO et al., 2008; GOULART et al., 2011) que são determinantes na identificação filogenética e mesmo taxonômica dos organismos presentes na biomassa microbiana e também na fauna do solo, e também pela maior sensibilidade desses organismos as alterações ocorridas no ambiente ao longo do espaço e do tempo.

Os estudos com organismos do solo da macro e meso fauna geralmente trabalham com levantamento de espécies, avaliando riqueza, abundância, diversidade e características funcionais e a possibilidade desses organismos servirem como indicadores de parâmetros físico-químicos de solo como nível de aeração, estruturação de agregados e fertilidade (MELO et al., 2009).

No contexto contemporâneo da pesquisa científica, não é possível que os estudos, tanto em conservação de ecossistemas quanto na ciência do solo, possam evoluir negligenciando as especificidades biológicas e ecológicas dos solos, das interações ecológicas entre plantas, herbívoros e seus inimigos naturais e todos os processos associados às interações abaixo e acima do solo. Precisamos conhecer melhor o solo, saber quem vive nele, como vive, porque e a que custo esses organismos se adaptaram a viver nesse ecossistema. Demandas para pesquisas nessa área são muitas. Não há como se pensar a conservação de maneira geossistêmica se todas as ciências não se dispuserem a colaborar com a tarefa de dar suas contribuições na produção de conhecimento sobre os processos de inter-relação que regem o equilíbrio sistemático da biodiversidade, sendo o solo a base principal de todo esse equilíbrio.

\section{CONSIDERAÇÕES FINAIS}

A ciência do solo se confunde com a própria história da humanidade, afinal o desenvolvimento das sociedades em muito tem a ver com a evolução das técnicas de manejo

Cultura Agronômica, Ilha Solteira, v.27, n.2, p.205-216, 2018 
e uso do solo, que por sua vez foram dando as configurações do processo de ocupação em cada pedoambiente no globo. Desse modo, é preciso dar a ciência do solo uma importância singular no mundo do conhecimento, pois todo o nosso desenvolvimento científico e tecnológico, urbano e industrial só foi possível porque havia alguém no campo aprendendo a lidar com os solos para produzir alimentos que pudessem sustentar toda a cadeia alimentar que mantém essas pessoas todas em suas atividades produtivas.

Por outro lado, o solo é um dos principais condicionadores de biodiversidade, pois atua na formação de distintos microambientes que resultam na diversidade de espécies. Podemos concluir então que o solo é a base de sustentação de tudo o que queremos conservar em termos de produção e ecologia vegetal. A ciência do solo é um dos saberes mais antigos que temos, apesar de tantas lacunas no conhecimento.

Já sabemos muito sobre o solo. Certamente o que nos falta para conseguir manejá-lo melhor é a integração de todos esses conhecimentos. Há vários caminhos a serem apontados: a formação de grupos de trabalho para pesquisas em redes, com diferentes enfoques, mas atuando em integração; política pública, de assistência técnica e extensão rural, atuando na promoção de técnicas de cultivo sustentáveis, formação de grupos multiprofissionais em órgãos de pesquisa públicos e privados, conjunto de instituições que atuam tanto no setor da agricultura quanto os que atuam na conservação da biodiversidade e mais integração entre os conteúdos curriculares de formação acadêmica. Os caminhos possíveis são muitos, as dificuldades em cada um deles existem, mas a necessidade da integração desses conhecimentos é superior a todos os desafios.

\section{REFERÊNCIAS BIBLIOGRÁFICAS}

ANTONIOLLI, Z. I.; CONCEIÇÃO, P. C.; BÖCK, V.; PORT, O.; SILVA, D. M.; SILVA, R. F. Método alternativo para estudar a fauna do solo. Revista Ciência Florestal, Santa Maria, v. 16, n. 4, p.407-417, 2006.

BARETTA, D.; FERREIRA, C. S.; SOUSA, J. P.; CARDOSO, E. J. B. N. Colêmbolos (Hexapoda: Collembola) como bioindicadores de qualidade do solo em áreas com Araucaria angustifólia. Revista Brasileira de Ciência do Solo, Viçosa, v. 32, n. especial, p.2694- 700, 2008.

BLOMQVIST, M. M.; OLFF, H.; BLAAUW, M. B.; BONGERS, T.; VAN DER PUTTEN, W. H. Interactions between above- and belowground biota: importance for small-scale vegetation mosaics in a grassland ecosystem. Oikos, Copenhagen, v. 90, n. 3, p.582-598, 2000 .

BORN, J.; PlUES, A. R.; BURSLEM, D. F. R. P.; NILU, R.; MAYCOC, C. R.; GHAZOUL, J. Differing Life History Characteristics Support Coexistence of Tree Soil Generalist and Specialist Species in Tropical Rain Forests. Biotropica, Lawrence, Kansas, v. 46, n. 1, p.58-68, 2014. 
CAVAlCANTI, L. F.; LIMA, M. F.; MEDEIROS, R. C. S.; MEGUERDITCHIAN, I. (Orgs.) Plano de Ação Nacional para a Conservação do Patrimônio Espeleológico nas Áreas Carsticas da Bacia do rio São Francisco. Brasília: Instituto Chico Mendes de Conservação da Biodiversidade, Instituto Chico Mendes, 2012. (Série Espécies Ameaçadas $\left.\mathrm{n}^{\mathrm{o}} 27\right) .140 \mathrm{p}$.

FIGUEIREDO, M. V. B.; BURITY, H. A.; STAMFORD, N. P.; SANTOS, C. E. R. S. Microorganismos e agrobiodiversidade: o novo desafio para agricultura. Guaíba: Agrolivros, 2008. 566 p.

FUGUEIREDO, M. V. B.; LIRA-JUNIOR, M. A.; ARAÚJO, A. S. F.; MARTINEZ, C. R. Fatores bióticos e abióticos à fixação biológica de $\mathrm{N}_{2}$. In: FUGUEIREDO, M. V. B.; BURITY, H. A.; STAMFORD, N. P.; SANTOS, C. E. R.S. Microorganismos e agrobiodiversidade: o novo desafio para agricultura. Guaíba: Agrolivros, 2008. Parte 1. cap. 2, p. 43-69.

FUKAMI, T.; WARDLE, D. A.; BELlinghAM, P. J.; MULDER, C. P. H.; TOWNS, D. R.; YEATES, G. W.; BONNER, K. I.; DURRETT, M. S. GRANT-HOFFMAN, M. N.; WILLIAMSON, W. M. Above- and below-ground impacts of introduced predators in seabird-dominated island ecosystems. Ecology Letters, University of Oxford, United Kingdom, v. 9, n. 12, p.1299-1307, 2006.

GIONGO, A.; BENEDUZI, A.; AMBROSINI, A.; VARGAS, L. K.; STROSCHEIN, M. R.; ELTZ, F. L.; BODANESE-ZANETTINI, M. H.; PASSAGLIA, L. M. P. Isolation and characteration of two plant growth-promoting bactéria from the rhizoplane of a legume (Lupinus albescens) in sandy soil. Revista Brasileira de Ciência do Solo, Viçosa-MG, v. 34, n. 2, p.361-369, 2010.

GIRACCA, E. M. N.; ANTONIOLLI, Z. I.; ELTZ, F. L. F.; BENEDETTI, E.; LASTA, E.; VENTURINI, S. F.; VENTURINI, E. F.; BENEDETTI, T. Levantamento da meso e macrofauna do solo na microbacia de Arroio Lino, Agudo/RS. Revista Brasileira de Agrociência, Pelotas, v. 9, n. 3, p.257-261, 2003.

GOULART, I. C. G. R.; MEROTTO JUNIOR, A.; NUNES, A. L.; BERED, F. Otimização da utilização de marcadores moleculares microssatélites e sua aplicação em estudos com plantas daninhas. Revista Planta Daninha, Viçosa, v. 29, n. especial, p.1175-1181, 2011.

HEIL, M. Plant-mediated interactions between above and below-ground communities at multiple trophic levels. Journal of Ecology, London, v. 99, n. 1, p.3-6, 2011.

LEPSCH, I. F. Formação e conservação dos solos. São Paulo: Oficina de Textos, 2002. $216 \mathrm{p}$.

MARTINS, C. M.; GALINDO, I. C. L.; SOUZA, E. R. S.; POROCA, H. A. Atributos químicos e microbianos do solo de áreas em processo de desertificação no semiárido de Pernambuco. Revista Brasileira de Ciência do Solo, Viçosa, v. 34, n. 6, p.1884-1890, 2010.

Cultura Agronômica, Ilha Solteira, v.27, n.2, p.205-216, 2018 
MCPHEE, C. S.; AARSSEN, L. W. The separation of above and below-ground competition in plants - A review and critique of methodology. Plant Ecology, Austrália, v. 152, n. 2, p.119-136, 2001.

MOÇO, M. K. S.; GAMA-RODRIGUES, E. F.; GAMA-RODRIGUES, A. C.; CORREIA, M. E. F. Caracterização da fauna edáfica em diferentes coberturas vegetais na região norte fluminense. Revista Brasileira de Ciência do Solo, Viçosa, v. 29, n. 4, p.556-564, 2005.

MOREIRA, F. M. S.; CARES, J. E.; ZANETTI, R.; STURMES, S. L. Ecossistema solo componentes, relações ecológicas e efeitos na produção vegetal. Lavras: UFL, 2013. 351 p.

MUGNAINI, R; STREHL, L. Recuperação e impacto da produção científica na era Google: uma análise comparativa entre o Google Acadêmico e a Web of Science. Revista Eletrônica de Biblioteconomia e Ciência da Informação, Florianópolis, n. especial, p.92$105,2008$.

PERSSON, H. (Org.) Above and below-ground interactions in forest trees in acidified soils. Uppsala, Sweden: Commission of the European Communities, 1990. 264 p.

PIZANO, C.; MANGAN, S. A.; HERRE, E. A.; EOM, A.; DALlinG, J. W. Above- and belowground interactions drive habitat segregation between two cryptic species of tropical trees. Ecology, Washington, v. 92, n. 1, p.47-56, 2011.

ROVEDDER, A. P. M.; ELTZ, F. L. F.; DRESCHER, M. S.; SCHENATO, R. B.; ANTONIOLLI, Z. I. Organismos edáficos como bioindicadores da recuperação de solos degradados por arenização no Bioma Pampa. Revista Ciência Rural, Santa Maria, v. 39, n. 4, p.1051-1058, 2009.

SINGH, R. P.; ONG, C. K.; SAHARAN, N. Above and below ground interactions in alleycropping in semi-arid India. Agroforestry systems, Honduras, v. 9, n. 3, p.259-274, 1989.

SILVA, R. R.; SILVA, M. L. N.; CARDOSO, E. L.; MOREIRA, F. M. S.; CURI, N. ALOVISI, A. M. T. Biomassa e atividade microbiana em solo sob diferentes sistemas de manejo na região fisiográfica campo das vertentes - MG. Revista Brasileira de Ciência do Solo, Viçosa, v. 34, n. 5, p.1586- 1592, 2010.

SOLER, R.; BEZEMER, T. M.; PUTTEN, W. H. V. B.; VET, L. E. M.; HARVEY, J. A. Root herbivore effects on above-ground herbivore, parasitoid and hyperparasitoid performance via changes in plant quality. Journal of Animal Ecology, London, v. 74, n. 6, p.1121-1130, 2005.

SOUTO, P. C.; SOUTO, J. S.; MIRANDA, J. R. P.; SANTOS, R. V.; ALVES, A. R. C. Comunidade microbiana e mesofauna edáficas em solo sob caatinga no semiárido da Paraíba. Revista Brasileira de Ciência do Solo, Viçosa, v. 32, n. 1, p.152-160, 2008.

STEVNBAK, K.; SCHERBER, C.; GLADBACH, D.J.; BEIER, C.; MIKKELSEN, T.N.; CHRISTENSEN, S. Interactions between above- and belowground organisms modified in

Cultura Agronômica, Ilha Solteira, v.27, n.2, p.205-216, 2018 
climate change experiments. Nature Climate Change, Londres, v. 2, n. 11, p.805-808, 2012.

VASCONCELOS, M. C. C. A.; SILVA, A. F. A.; LIMA, R. S. Cultivo em aléias: uma alternativa para pequenos agricultores. Revista Agropecuária Científica no Semiárido, Campina Grande, v. 8, n. 3, p.18-21, 2012.

VAZ-DE-MELO, F.; BROWN, G. G.; CONSTANTINO, R.; LOUZADA, J. N. C.; LUIZÃO, F. J.; MORAIS, J. W.; ZANETTI, R. A importância da meso e macrofauna do solo na fertilidade e como bioindicadores. Boletim Informativo da Sociedade Brasileira de Ciência do Solo, Viçosa, v. 34, n. 1, p.39-43, 2009.

VEZZANI, F. M.; MIELNICZUK, J. Uma visão sobre qualidade do solo. Revista Brasileira de Ciência do Solo, Viçosa, v. 33, n. 4, p.743-745, 2009.

WACKERS, F.; BEZEMER, T. M. Root herbivory induces an above-ground indirect defence. Ecology Letters, Oxford , v. 6, n. 1, p.9-12, 2003.

WARDLE, D. A. The influence of biotic interactions on soil biodiversity. Ecology Letters, Oxford, v. 9, n. 7, p.870-886, 2006.

ZILLI, J. E; RUMJANEK, N. G.; XAVIER, G. R.; COUTINHO, H. L. C.; NEVES, M. C. P. Diversidade microbiana como indicador de qualidade de solo. Cadernos de Ciência e Tecnologia, Brasília, v. 20, n. 3, p.391-411, 2003. 